\title{
다(1) $(5)$
}

\section{El engagement y la sorpresa en la comunicación digital de las marcas}

Estudio del impacto emocional de los GIFs en los usuarios de las redes sociales

\section{Engagement and the Element of Surprise in Digital Communication of Brands A study of the Emotional Impact of GIFs in Social Network Users}

Idoia Rúa Hidalgo

Laboratorio Neuromarketing de la Universidad Internacional de La Rioja. idoia.rua@gmail.com https://orcid.org/0000-0002-0497-8001

Dra. María Galmes-Cerezo

Profesora Dept. Organizacion de Empresas y

Marketing. Universidad Complutense de Madrid mgalmes@ucm.es

https://orcid.org/0000-0002-1520-3703

María del Cisne Espinosa Jarrín Laboratorio Neuromarketing de la Universidad Internacional de La Rioja. maricis.e@gmail.com https://orcid.org/0000-0001-7580-0094
Rúa Hidalgo, I., Galmes-Cerezo, M. y Espinosa Jarrín, M. (2021)

El engagement y la sorpresa en la comunicación digital de las marcas. Estudio del impacto emocional de los GIFs en los usuarios de las redes sociales

Revista Internacional de Investigación en Comunicación aDResearch ESIC. NN 25 Vol 25

Monográfico especial, marzo 2021 · Págs. 26 a 43

https://doi.org/10.7263/adresic-025-02 
RESUMEN

\section{Clasificación JEL: \\ M3}

\section{Palabras clave:}

Comunicación

digital,

GIFs,

Emociones,

Engagement,

Sorpresa,

Marcas,

Neuromarketing,

Asociaciones

implícitas
Objetivo: Se han realizado dos estudios. El objetivo principal del estudio 1 ha sido conocer los efectos emocionales y el engagement que los recursos de comunicación «GIFs» provocan en los usuarios. Como objetivo principal del estudio 2 se ha buscado determinar las actitudes implícitas que tienen los participantes respecto a las marcas muy conocidas.

Diseño metodológico: En el primer estudio se utiliza una combinación de herramientas biométricas de observación automatizada de las expresiones faciales, conductancia de la piel y dirección de la pupila, para observar el estado emocional que los GIFs de marcas renombradas provocan en los participantes, y compararlo con los efectos causados por imágenes estáticas de estas mismas marcas. El segundo estudio emplea el Test de asociación implícita para observar asociaciones inconscientes relacionadas con marcas conocidas.

Resultados: Los resultados del primer estudio muestran que los GIFs consiguen el engagement de los usuarios y provocan un «estado de bienestar-placer» (Modelo Circumplejo de Russel, 1980), pero no el estado entusiasta que se esperaba. Los resultados del segundo estudio indican que los participantes tienen la creencia de que las marcas muy conocidas son marcas de calidad, mientras que no asocian este atributo a las marcas no conocidas. La correlación encontrada entre los resultados de los dos estudios revela que los GIFs, aun provocando emociones positivas y generando engagement, no consiguen un estado entusiasta, cuando las marcas son interiorizadas como marcas de calidad.

Limitaciones e implicaciones: El método de investigación utilizado ha mostrado su utilidad para medir el engagement de los usuarios en la visualización de los GIFs utilizando métricas como el signo y la intensidad de la emoción, y herramientas de neuromarketing. El estudio experimental ha tenido su principal limitación en no poder observar los efectos emocionales que generan los GIFs que permiten interacción del usuario

Originalidad y contribución: Las marcas conocidas, además de utilizar estos recursos visuales innovadores, tienen que usarlos de forma diferente para generar sorpresa y conseguir situar a los clientes potenciales en el «estado emocional entusiasta» del Modelo Circumplejo (Russel,1980).

\section{ABSTRACT}

\section{JEL Classification: \\ M3}

Key words:

Digital communication, GIFs, emotions, engagement, surprise, brands, neuromarketing, implicit associations
Purpose: Two studies were carried out. The main objective of study 1 was to find out the emotional effects and engagement that «GIFs» communication resources provoke in users. The main objective of study 2 was to determine the implicit attitudes that participants have towards well-known brands.

Design / Methodology /Approach: The first study uses a combination of biometric tools of automated observation of facial expressions, skin conductance and pupil direction to observe the emotional state that GIFs of well-known brands elicit in participants, and compare it to the effects caused by static images of the same brands.

The second study uses the Implicit Association Test to observe unconscious associations related to well-known brands.

Results: The results of the first study show that GIFs achieve user engagement and provoke a «feel-good-pleasure state» (Russel's Circumplex Model, 1980), but not the expected enthusiastic state. The results of the second study indicate that participants have the belief that wellknown brands are quality brands, while they do not associate this attribute with non-famous brands. The correlation found between the results of the two studies reveals that GIFs, while provoking positive emotions and generating engagement, do not achieve an enthusiastic state when brands are internalised as quality brands.

Limitations / implications: The research method used has shown its usefulness in measuring user engagement in viewing GIFs using metrics such as the sign and intensity of emotion, and neuromarketing tools. The experimental study had its main limitation in not being able to observe the emotional effects generated by GIFs that allow user interaction.

Originality / contribution: Well-known brands, in addition to using these innovative visual resources, have to use them in a different way to generate surprise and manage to place potential customers in the «enthusiastic emotional state» of the Circumplex Model (Russel,1980). 


\section{Introducción ${ }^{1 \text { y } 2}$}

La comunicación de marketing actual demanda nuevos desafíos ante la presencia de usuarios hiperconectados, sobre-estimulados y saturados de información (Estrella y Segovia, 2016). Por ello seleccionar los recursos comunicacionales a emplear resulta cada vez más necesario (Adami y Jewitt, 2016).

La comunicación digital asume hoy en día una postura protagonista en la comunicación de las marcas (Powers et al., 2012; Scolari, 2012). El entorno digital es cada vez más visual y las empresas, con el propósito de captar la atención de los usuarios, utilizan en las redes sociales recursos visuales creativos y atractivos para comunicar sus marcas, ofertar sus productos y seducir a sus clientes (Zhang y Mao, 2016).
El uso de los GIF puede desencadenar percepciones inconscientes del usuario, a través de las neuronas espejo (Likowski et al., 2012; Cacioppo, Bolmont y Monteleone, 2018). Esta estimulación sensorial ofrece facilidad cognitiva al receptor apelando a su respuesta automática (Kahneman y Tversky, 1979). Los GIFs ayudan a la representación mental en el usuario (Holmes et al., 2008), y al estar en movimiento, representan una acción que está enviando comunicación no verbal y creando significación (Tolins y Samermit, 2016). Son cada vez más utilizados en las estrategias de comunicación digital por su simplicidad, fácil representación y capacidad de provocar empatía con el contenido mostrado (Duffett, 2015; Miltner y Highfield, 2017).

\section{Figura 1. Metodología estudio 1 y estudio 2}

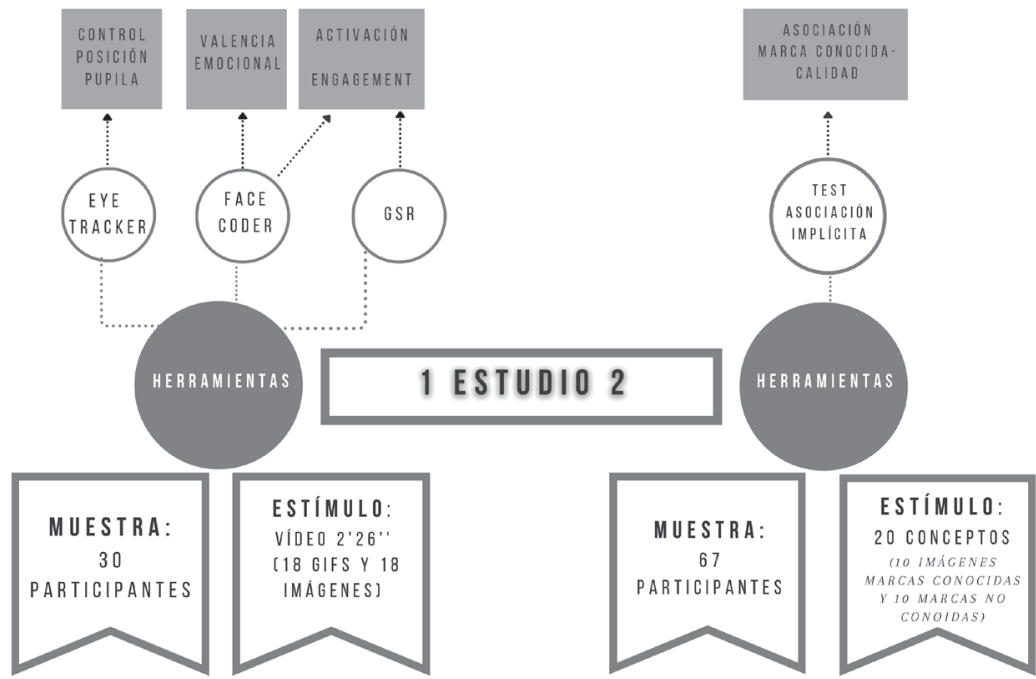

Fuente: Elaboración propia.

1 Graphics Interchange Format (GIF) El GIF es un archivo de imágenes gráficas en un mapa de bits que incorpora animación a las imágenes. Se utiliza fundamentalmente en internet y redes sociales.

2 Agradeciemientos al apoyo prestado por la empresa INTERACTUA+ y por el Laboratorio de neuromarketing de la Universidad Internacional de la Rioja 


\section{Estudio 1}

\subsection{Los GIFs como recursos favorecedores de una experiencia de marca}

Los GIFs, como elementos de comunicación digital, facilitan la comprensión del mensaje a través de la empatía. Ofrecen al receptor una experiencia, a partir de la cual los usuarios construirán sus percepciones sobre las marcas (Novak, Hoffman y Yung, 2000). Las experiencias comienzan siendo algo externo, diseñado por la marca, y una vez vividas se convierten en algo interno para el usuario (Yuang y Wu, 2008; Skandalis, Byrom y Banister, 2019). Las marcas utilizan GIFs para diseñar experiencias con cualidades afectivas (Gürsimsek, 2016) y sensoriales convirtiéndose así, en un excelente proveedor de la experiencia de marca (Brakus, Schmitt y Zarantonello, 2009).

Las experiencias de marca generadas por un GIF, para ser significativas, deben ser memorables, satisfactorias y que conecten con las emociones (Schmitt, 1999; Galmés, 2015; Atwal y Williams, 2017). Fomentar emociones en el usuario incrementa la satisfacción y motiva al usuario a realizar una acción determinada (González-Sánchez y Gil-Iranzo, 2013). Una experiencia de marca tiene como resultado una respuesta emocional de placer o displacer que surge a partir de la activación emocional (FernándezAbascal et al., 2010).

\subsection{Los GIFs proveedores de emociones}

Hay estudios que muestran que los GIFs son un recurso que actúa como detonante emocional en el cerebro del receptor durante su visualización (Jou, Bhattacharya y Chang, 2014; Tianliang et al., 2019). Las imágenes animadas tienen mayor capacidad de engagement en los usuarios que las imágenes estáticas (Chang y Unga, 1993; McKenna et al., 2017). Por ello, las marcas están utilizando los GIFs en sus estrategias de comu- nicación online consiguiendo generar altos niveles de engagement emocional (Cingel y Krcmar, 2014; Calder, Malthouse y Moslowska, 2016; Thurlow, Aiello y Portmann, 2019).

Considerando la relación existente entre la visualización de los GIFs y la respuesta emocional, según el Sistema Internacional de Imágenes Afectivas (IAPS), que estudia la emoción y la atención provocada ante estímulos visuales (Vila, Ramírez, Sánchez y Fernández-Santaella, 2001), la emoción puede sugerir una predisposición a la acción. Esta predisposición resulta de la activación de determinados circuitos cerebrales ante estímulos significativos. Cuando estos circuitos se activan provocan reacciones fisiológicas, conductuales y emocionales que son guiadas por patrones específicos. (Enli, 2017).

Los GIFs podrían actuar como desencadenantes emocionales no permitiendo la adaptación hedónica en el individuo (Ryan y Deci, 2001), sino manteniéndole activo ante su exposición, a través de la sorpresa que se produce de forma rápida mediante sensaciones placenteras espontáneas e inesperadas. (Izard, 1991).

Algunos estudios han demostrado que un mayor engagement y una mayor activación emocional indican una tendencia a la movilización hacia la compra (Brodie et al., 2011; Hollebeek, 2011).

\subsection{Hipótesis del Estudio 1}

A partir de la revisión de la literatura científica, se plantean las siguientes hipótesis:

- $\mathrm{H}_{1}$ Los participantes tendrán emociones positivas (placer) al visualizar los GIFs.

- $\mathrm{H}_{2}$ : Los participantes tendrán emociones más positivas (placer) al visualizar los GIFs que al visualizar las imágenes estáticas.

- H3: Los participantes tendrán emociones positivas e intensas (engagement) al visualizar los GIFs. 
- H4: Los participantes tendrán más emociones positivas de mayor intensidad (engagement) al visualizar los GIFs que al visualizar las imágenes estáticas.

- H5: Los participantes tendrán activación positiva (orientación a la acción) al visualizar los GIFs.

- H6: Los participantes tendrán una mayor activación al visualizar los GIFs que al visualizar las imágenes estáticas.

\subsection{Materiales y Métodos del Estudio 1}

Se ha realizado una investigación experimental con una combinación de tres herramientas de análisis biométrico: Face coder, Eye tracker y Skin conductance. La aplicación de las herramientas Face coder - para la codificación de las emociones registradas - y Eye tracker — para el seguimiento ocular - ha medido la Valencia de las emociones registradas y el nivel de Engagement obtenido. Paralelamente y de forma sincronizada, los participantes fueron monitorizados a través de la herramienta Skin conductance ${ }^{3}$, obteniéndose el nivel de Arousal durante la visualización. Se hace uso de las herramientas biométricas Face Coder y Eye Tracker de la empresa INTERACTÚA +, cedidas por el Laboratorio de Neuromarketing de la Universidad Internacional de la Rioja y la herramienta de Skin Conductance de la marca Esense (Tabla 1).

La selección de los participantes se ha realizado de forma aleatoria, con el único requisito de utilizar habitualmente al menos una red social. El tamaño de la muestra de 30 participantes válidos se considera adecuado para estudios experimentales que utilizan las mismas herramientas biométricas (Ekman, Freisen, y Ancoli, 1980; Vechiato, et al., 2010; Tapia Frade, et al., 2016) y, que como indican Monge-Benito y Fernández-Guerra (2011), ofrecen un margen de error del 1 \%. Para la distribución de la muestra se utilizó el estudio Interactive Advertising Bureau y Elogia (2018), que define los perfiles de usuarios de redes sociales, y su composición es la siguiente: 33 \% para cada rango de edad (16-30; 31-45; 46-55), con un $53 \%$ de mujeres y un $47 \%$ de hombres.

El estímulo utilizado en la investigación experimental fue un vídeo de 2 minutos 26 segundos, formado por la combinación aleatoria de 18 GIFs

\section{Tabla 1. Herramientas del Estudio}

\begin{tabular}{l|l}
\hline Instrumento y antecedentes empíricos & Tipo de registro \\
\hline Eye Tracker (Chang y Chen, 2017; Audrin et al., 2018). & $\begin{array}{l}\text { Seguidor ocular. Registra la mirada del sujeto mientras está } \\
\text { visualizando un estímulo. Registra la posición del ojo y orientación } \\
\text { de la cabeza y proporciona información de lo que el sujeto está } \\
\text { visualizando }\end{array}$ \\
\hline $\begin{array}{l}\text { Face Coder (McDuff, El Kaliouby y Picard, 2012; Bellman, } \\
\text { Wooley y Varan, 2016; Goyal y Singh, 2018; Mundel et al, 2018). }\end{array}$ & $\begin{array}{l}\text { Decodificación del rostro de la persona a través de un software } \\
\text { que analiza la imagen proporcionada por la cámara web. Se } \\
\text { obtienen } 6 \text { posibles emociones (alegría, asco, tristeza, ira, sorpresa } \\
\text { y miedo), las } 6 \text { emociones básicas descritas por Ekman. }\end{array}$ \\
\hline $\begin{array}{l}\text { GSR Galvanic Skin Conductance (Weibel et al, 2019; Walla, } \\
\text { Koller, Brenner y Bosshard, 2017; Guerreiro, Rita y Trigueiros, } \\
\text { 2015; Reimann, Castano, Zaichkowsky y Bechara; 2012). }\end{array}$ & $\begin{array}{l}\text { Medición de la conductancia de la piel a través de electrodos colo- } \\
\text { cados en los dedos de la mano del sujeto. Proporciona información } \\
\text { sobre procesos inconscientes del sujeto ante la exposición a un } \\
\text { estímulo. }\end{array}$ \\
\hline
\end{tabular}

3 Arousal: El constructo arousal (activación) es un término hipotético que describe los procesos que controlan la alerta, la vigilia y la activación (Anderson, 1990). 
y 18 imágenes estáticas de entre 2 y 4 segundos por elemento para evitar un procesamiento consciente. Se han seleccionado 18 GIFs y 18 imágenes estáticas de entre las 100 mejores marcas a nivel mundial según el Ranking anual Best Global Brands 2017 (Interbrand, 2017). Se escogieron los GIFs de estas marcas que mayor número de «me gusta» habían tenido en Instagram (1 marzo. - 10 abril 2019), y la imagen estática correspondiente extraída del GIF. Para evitar sesgos de presentación, se han editado tres vídeos distintos con los elementos ordenados aleatoriamente.

Todos los sujetos fueron expuestos al visionado del estímulo en alguna de las tres versiones. Al mismo tiempo que se registraron las microexpresiones faciales (Ekman,1993), se midió el nivel de conductancia de la piel, obteniéndose de este modo la intensidad de la emoción.

Se han registrado tres variables: Activación emocional, Valencia emocional y Engagement. Todas las variables dependientes ofrecen la medida para cada sujeto y cada GIF e imagen está- tica, y estos datos individuales se añaden a los valores agregados del grupo, obteniendo así un valor cuantitativo de cada variable por cada GIF e imagen estática. La variable Valencia mide el signo de la emoción: positiva o negativa. La variable Activación registra la intensidad de la emoción, que se obtiene midiendo el tipo de emoción registrada (activante o desactivante) y las variaciones del nivel de sudoración de la piel. La variable Engagement indica el estado emocional en el que se encuentra la persona cuando visiona cada GIF e imagen estática y se extrae de la combinación de la Valencia emocional y la Activación (Tabla 2).

\subsection{Resultados y discusión Estudio 1}

La medida de todas las variables analizadas resultó superior para los GIFs que para las imágenes (Valencia: $\mathrm{MGIF}=14.809>\mathrm{MIMG}=14.61$; Activación: MGIF $=-14.46>$ MIMG $=-14.54 ;$ Engagement: $\mathrm{MGIF}=.248>\mathrm{MIMG}=.05$ ). Los resultados se muestran en la tabla 3.

\section{Tabla 2. Variables, Dimensiones e Instrumentos}

\begin{tabular}{|l|l|l|}
\hline Variable & Dimensión & Herramienta \\
\hline Activación & Intensidad de la emoción & Face coder+GSR \\
\hline Valencia Emocional & Signo de la emoción & Face coder \\
\hline Engagement (Valencia + Activación) & Estado emocional & Facecoder+GSR \\
\hline
\end{tabular}

Tabla 3. Resultados de las variables dependientes

\begin{tabular}{|c|c|c|c|c|c|}
\hline & $\begin{array}{l}\text { Tipo } \\
\text { Recurso }\end{array}$ & $\mathbf{N}$ & Media & Desviación tip. & Error promedio \\
\hline \multirow{2}{*}{ Valence } & Imagen & 30 & 14,611667 & 3,4733646 & 8186799 \\
\hline & GIF & 30 & 14,809444 & 3,5661917 & ,8405594 \\
\hline \multirow{2}{*}{ Activation } & Imagen & 30 & $-14,544444$ & 2,0190861 &, 4759031 \\
\hline & GIF & 30 & $-14,463333$ & 2,3245265 & ,5478962 \\
\hline \multirow{2}{*}{ Engagement } & Imagen & 30 & ,050000 & 3,1002030 & ,7307249 \\
\hline & GIF & 30 & 248333 & 3,0898910 & ,7282943 \\
\hline
\end{tabular}


Todas las variables (Valencia, Activación y Engagement) se aproximan a la normalidad tanto en el caso de las imágenes como para los recursos GIFs según la prueba de normalidad ShapiroWilk (Valencia: p>.05 PImagen=.651 y $\mathrm{P}_{\text {Gif }}=.779$; Activación: $\mathrm{p}>.05 \mathrm{P}_{\text {Imagen }}=.165$ y $\mathrm{P}_{\mathrm{Gil}}=.346$; Engagement: $\mathrm{p}>.05 \mathrm{P}_{\text {Imagen }}=.299$ y $\mathrm{P}_{\mathrm{Gi}_{\mathrm{f}}}=.772$ ), presentando sus varianzas en todos los casos homogeneidad, atendiendo a los resultados obtenidos en la prueba de Levene de igualdad de varianzas

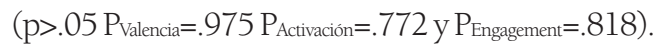

Dado que se cumple el criterio de normalidad y homocedastidad para todas las distribuciones de las variables cuantitativas, se utiliza la prueba t de Student para muestras independientes a fin de comparar las medias. La prueba T para igualdad de medias indica que no hay asociación entre la Valencia, la Activación o el Engagement y el tipo de recurso imagen estática o GIF. En todos los casos se aceptó la hipótesis nula de no diferencia significativa entre las medias de las variables continuas cuando se trata de imágenes o de GIFs (p>.05 PValencia=.867 PActivación=.912 y $\mathrm{P}_{\text {Engagement=.849). }}$ En las tres variables dependientes la media es mayor para los GIFs que para las imágenes estáticas, aunque no significativamente superior.

Para las variables Valencia y Engagement las medias presentan un valor positivo. Sin embargo, la Activación presenta una media de signo negativo.

Atendiendo a los resultados obtenidos el contraste de las hipótesis sería el siguiente:

- $\mathrm{H}_{1}$ : La media de la variable Valencia ante los GIFs ha resultado positiva, por lo que se confirma la hipótesis de que los participantes tienen emociones positivas al visualizar los GIFs.

- $\mathrm{H}_{2}$ : La media de la variable Valencia ante los GIFs ha sido superior a la de las imágenes estáticas, pero teniendo en cuenta que la di- ferencia no ha sido estadísticamente significativa, no es posible confirmar la hipótesis de que los participantes tienen emociones más positivas al visualizar los GIFs que al visualizar las imágenes estáticas.

- $\mathrm{H}_{3}$ : La media de la variable Engagement ante los GIFs ha resultado positiva, por lo que se confirma la hipótesis de que los participantes han tenido emociones positivas e intensas al visualizar los GIFs.

- $\mathrm{H}_{4}$ : La media de la variable Engagement ante los GIFs ha sido superior a la de las imágenes estáticas, pero teniendo en cuenta que la diferencia no ha sido estadísticamente significativa, no es posible confirmar la hipótesis de que los participantes han tenido más emociones positivas y de mayor intensidad al visualizar los GIFs que al visualizar las imágenes estáticas.

- $\mathrm{H}_{5}$ : La media de la variable Activación ante los GIFs es de signo negativo. Por tanto, se refuta la hipótesis de que los participantes estarán activados hacia la compra al visualizar los GIFs.

- H6: La media de la variable Activación ha resultado superior para los GIFs que para las imágenes estáticas, pero al no ser la diferencia estadísticamente significativa no se puede confirmar la hipótesis de que los participantes están más activados al visualizar los GIFs que al visualizar las imágenes estáticas.

La correlación inversa encontrada entre la variable Valencia y la variable Activación nos ha llevado a preguntarnos cuál podría ser la causa de que los GIFs estén generando en los consumidores sensación de bienestar (Valencia positiva), y al mismo tiempo estén provocando una Activación negativa. Se plantea un segundo estudio con el objetivo de explicarlo. 


\section{Estudio 2}

\subsection{Marcas conocidas, calidad percibida y emociones hedónicas}

El concepto de Valor de marca (brand equity) hace referencia al valor que los consumidores atribuyen a una marca. Aaker fue pionero en identificar unas dimensiones para calcular el valor de las marcas: el conocimiento de la marca, las asociaciones a la marca, la calidad percibida y la fidelidad (Aaker, 1991).

La calidad percibida es la evaluación subjetiva de la excelencia de una marca por parte de los consumidores. Para realizar esta evaluación, las personas utilizan indicios externos y asociaciones internas (Aaker, 1996; Klaus y Maklan, 2007). Los consumidores no pueden hacer juicios completos y absolutamente objetivos sobre la calidad objetiva de una marca y utilizan asociaciones para inferir calidad a una marca o producto (Ophuis y Van Trijp, 1995).

Aaker (1991) mostró la existencia de relación directa entre una marca bien conocida y la calidad percibida y estudios posteriores, que investigan el Valor de marca, han confirmado que los consumidores tienden a asociar calidad a las marcas que conocen (Yoo y Donthu, 2001; Pawle y Cooper, 2006). Un mayor conocimiento de la marca y unas asociaciones de marca más fuertes conducen a una mayor calidad percibida (Lavidge y Steiner, 1961; Ding y Tseng, 2015).

La emoción hedónica ha sido descrita como un estado mental que surge a partir de evaluaciones de eventos o pensamientos. Puede desencadenar acciones específicas para reafirmar o contrarrestar la emoción (Bagozzi et al., 1999). Los consumidores tienden a sentir emociones hedónicas positivas en el proceso de compra y de consumo (Hirschman y Holbrook, 1982; Havlena y Holbrook, 1986). Las estrategias de marketing experiencial tratan de provocar emociones hedó- nicas en los compradores, en los momentos en que se produce un contacto con la marca ya sea durante la compra, el consumo o en el proceso de comunicación (Galmés, 2012; Schmitt, Brakus, y Zarantonello, 2015). Cuando el consumidor siente que la marca puede ayudarle a alcanzar sus objetivos, se genera un estado placentero. Es así como una experiencia de la marca puede servir como un evento subjetivo que provoca una valoración positiva de la marca por parte de los consumidores. (Zeithaml, 1988; Ding y Tseng, 2015)

\subsection{Las emociones positivas son necesarias pero no suficientes para movilizar al usuario hacia la compra}

Lo que buscan las marcas es posicionarse en la mente y en el corazón de sus clientes y la involucración de los sentidos es fundamental para influir en su estado emocional (Prescott, 2017). La principal experiencia de valor es la emoción (Smith y Bolton, 2002). Cuando existe una respuesta emocional se detectan dimensiones como la Valencia y la Intensidad o Arousal (Lang, 1995). Para que las emociones deriven en una movilización hacia la compra, es necesario que estas no solamente sean de Valencia positiva, sino que además exista una Activación de signo positivo. Todo ello de acuerdo al Modelo Circumplejo de las emociones (Russell, 1980) que explica como la combinación de los valores Activación y Valencia nos permite diferenciar los distintos estados emocionales en los que se encontraría el usuario como consecuencia del estímulo presentado (Plutchik y Conte, 1997; Jaeger et al., 2018). (Véase figura 2 en pág. 34).

Las emociones positivas son más habituales que las negativas en los usuarios de redes sociales (He et al., 2016), y las experiencias emocionales del mundo real pueden transferirse al entorno 


\section{Figura 2 Estados emocionales según el Modelo Circumplejo. Russell (1980)}

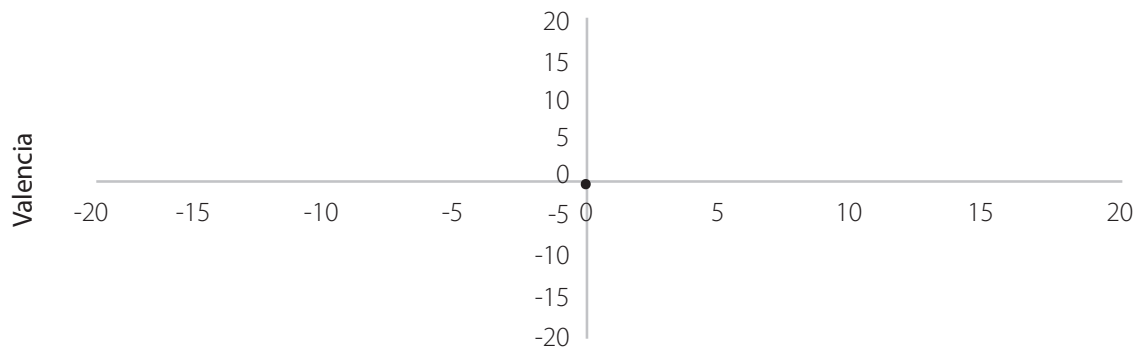

Activación

Fuente: Elaboración propia.

virtual (Kafetsios et al., 2017). Las emociones influyen en las decisiones del individuo a nivel personal o en su rol de consumidor (Burnett y Lunsford, 1994). Por ello, las marcas buscan puntos de contacto, como el GIF, para emocionar a sus clientes mediante las experiencias diseñadas provocando altos niveles de engagement que les movilice hacia la compra.

El valor que el consumidor atribuye a la marca conocida ${ }^{4}$, le lleva a anticipar el uso de recursos innovadores en sus estrategias de comunicación. Sin embargo, las marcas no conocidas por el usuario tienen una mayor probabilidad de provocar, a través de estímulos novedosos, una reacción de tipo afectiva inconsciente (Öhman, 1987; Godey et al., 2016). Es muy posible que las marcas conocidas estén en desventaja a la hora de provocar sorpresa en los usuarios.

\subsection{Hipótesis y preguntas de investigación} La revisión de la literatura académica ha servido para contextualizar una nueva hipótesis, y los

4 El concepto «marca conocida», en este artículo hace referencia a la idea de marca famosa o marca renombrada. resultados del estudio 1 han desencadenado una nueva pregunta de investigación:

- $\mathrm{H}_{7}$ : Los consumidores asocian de forma inconsciente las marcas conocidas con alta calidad y asocian las marcas no conocidas con menor calidad.

- RQ1: ¿Es posible explicar el estado emocional de los usuarios ante los GIFs de marcas conocidas, a partir de las correlaciones entre los resultados de las variables del Estudio 1 y la Variable $\mathrm{D}_{\text {(IAT) }}$

\subsection{Materiales y métodos del Estudio 2}

Se lleva a cabo un experimento con el Test de Asociación Implícita (IAT) con el fin de descubrir posibles asociaciones no conscientes en los sujetos entrevistados. Esta prueba, diseñada por los investigadores Greenwald y Banaji (1995), diagnostica las preferencias actitudinales que una persona tiene pero de las que no es plenamente consciente (Blanton y Jaccard 2006). Permite obtener una evaluación de la fuerza de las asociaciones automáticas. El IAT ha sido utilizado y testada su validez en otros estudios relacionados con la percepción implícita de las marcas (Chang, 
Ko y Carlson, 2018; Sharma, 2018; Vriens, Chen y Schomaker, 2019). Las asociaciones implícitas tienen su origen en la evaluación automática o inconsciente del cerebro. Se miden a través de la latencia o tiempo de respuesta ante la presentación de un estímulo. La prueba consiste en discriminar entre estímulos visuales y palabras con valencias positivas y negativas lo más rápidamente posible, detectando así la asociación entre conceptos basándose en el tiempo de reacción.

Los sujetos son los mismos participantes del estudio 1 y se han añadido 37 sujetos nuevos con el fin de obtener el tamaño muestral validado en otras investigaciones con IAT (Maison, Greenwald y Bruin, 2004; Gibson, 2008). La distribución según género y rango de edad es similar a la del estudio 1.

El estímulo ha consistido en 20 conceptos, compuestos por diez imágenes de marcas conocidas y diez de marcas blancas. Para la selección de las marcas conocidas se ha acudido al estudio Brand Footprint Report 2018 (Kantar Worldpanel, 2018) que elabora un ranking con las marcas mas populares. Del que se extrajeron las 10 mar- cas más elegidas en España. En concreto, se han utilizado las dos primeras marcas más populares de cada uno de los siguientes sectores «Alimentación», «Bebidas», «lácteos», «Droguería»e «Higiene y cuidado personal» (Figura3).

Estas marcas se asocian con 20 atributos: diez palabras relacionadas con el concepto «calidad» y diez con el concepto «ausencia de calidad». Se diseñan un total de veinte presentaciones combinadas por usuario y etapa, de acuerdo con la metodología descrita por Greenwald (Greenwald et al., 2003). Durante la prueba todos los sujetos cumplimentaron el test compuesto de las 5 etapas en las que se combinaron conceptos a través de imágenes y atributos mediante palabras. Todos los participantes fueron informados de que debían responder de forma rápida, pero sin cometer excesivos errores.

Se ha medido el tiempo de respuesta de cada participante calculando el lapso entre la presentación del estímulo y la elección de la respuesta. Esta medida ha servido para calcular «el efecto IAT» o D(IAT) para cada sujeto. La D (IAT) es el algoritmo que mide la latencia de respuesta y se

\section{Figura 3. Marcas conocidas incluidas en la investigación. Kantar Worldpanel, 2018}

\section{Top5 marcas por sectores}
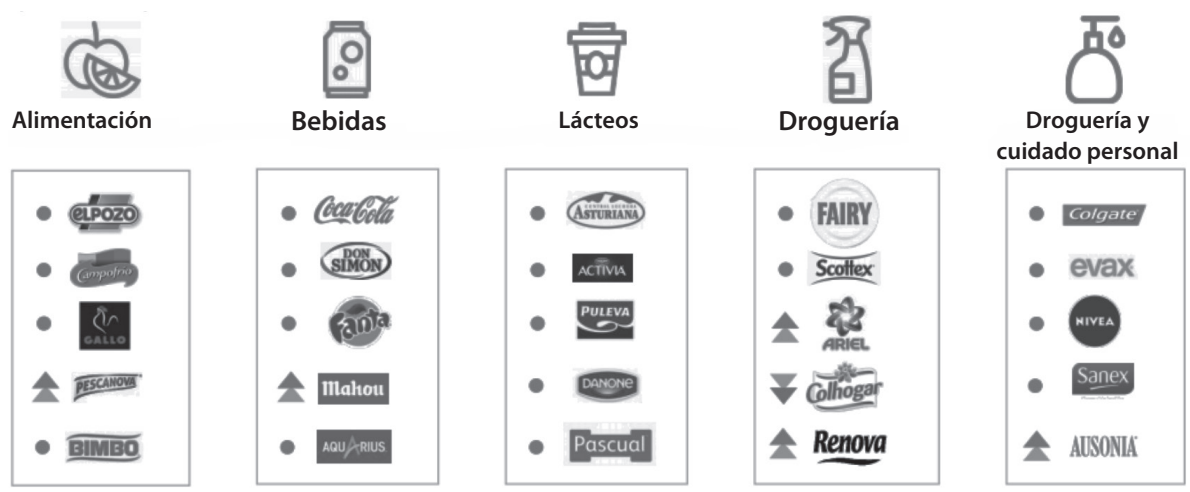
calcula como la diferencia entre el promedio de las velocidades de reacción (milisegundos) de los sujetos en cada bloque, dividido entre la desviación estándar de las latencias para cada bloque.

\subsection{Resultados y discusión Estudio 2}

El resultado obtenido en el Test de Asociación Implícita ( $\mathrm{D}_{(\mathrm{IAT})=.59)}$ indica una asociación moderada alta entre marca conocida y «calidad».

Para comprobar si existe una relación entre la variable DIAT y la emoción Sorpresa se ha aplicado el índice de correlación de Pearson. El resultado ha registrado una correlación significativa en el nivel .05 de signo negativo (-.437) que indica una relación inversa entre ambas variables. Por tanto, una mayor asociación im- plícita entre marca reconocida y calidad generaría una menor emoción de sorpresa en el consumidor (Tabla 4).

Por otra parte, al observar en el Estudio 1 que todos los GIFs analizados presentaban valores negativos para la variable Activación, se decide seguir buscando relaciones entre las variables emocionales que puedan explicar estos resultados. El coeficiente de correlación de Pearson para analizar si existe correlación entre la variable Activación y la Sorpresa, mostró una correlación elevada y significativa en el nivel .01 de signo positivo ( $\mathrm{r}=.601)$ entre ambas variables. Este resultado indica que cuanta mayor sorpresa genera un recurso en el usuario, mayor activación provoca en él (Tabla 5).

\section{Tabla 4. Correlación entre la variable $D_{I A T}$ (índice asociación implícita) y la emoción sorpresa}

\section{Correlaciones}

\begin{tabular}{|c|c|c|c|}
\hline & & Activación & Sorpresa \\
\hline \multirow{3}{*}{ IAT } & Correlación de Pearson & 1 &,$- 437^{*}$ \\
\hline & Sig. (bilateral) & &, 033 \\
\hline & N & $t^{*}$ & 30 \\
\hline \multirow{3}{*}{ Sorpresa } & Correlación de Pearson &,$- 437^{*}$ & 1 \\
\hline & Sig. (bilateral) & ,033 & \\
\hline & N & 30 & 30 \\
\hline
\end{tabular}

**. La correlación es significativa en el nivel 0,05 (bilateral).

\section{Tabla 5. Correlación entre la variable activación y la emoción sorpresa}

\section{Correlaciones}

\begin{tabular}{|c|c|c|c|}
\hline & & Activación & Sorpresa \\
\hline \multirow{3}{*}{ Activación } & Correlación de Pearson & 1 &, $601^{* *}$ \\
\hline & Sig. (bilateral) & &, 002 \\
\hline & N & $+^{*}$ & 30 \\
\hline \multirow{3}{*}{ Sorpresa } & Correlación de Pearson &, $601^{* *}$ & 1 \\
\hline & Sig. (bilateral) &, 002 & \\
\hline & N & 30 & 30 \\
\hline
\end{tabular}

**. La correlación es significativa en el nivel 0,01 (bilateral) 
Por tanto, la $\mathrm{H}_{7}$ se ha confirmado con el resultado del Test de Asociación implícita que muestra una asociación moderada-alta. Esto indica que los consumidores tienen una creencia inconsciente de que las marcas que conocen bien son marcas de calidad.

Respecto a la pregunta de investigación $\left(\mathrm{RQ}_{1}\right)$ en la que nos planteábamos la posibilidad de explicar el estado emocional de los usuarios ante los GIFs de marcas conocidas, efectivamente se puede responder a través de la correlación entre la variable Activación y la variable Sorpresa; y a partir de la correlación entre la variable Sorpresa y la variable DIAT,

- La existencia de una correlación positiva entre la variable Activación y la emoción Sorpresa explica que las marcas, para conseguir activar a los consumidores, tienen que sorprenderles. Los GIFs analizados, a pesar de ser un recurso diferente, no logran generar sorpresa y por ello, el estado emocional conseguido es de bienestar-placer (cuarto cuadrante del modelo circumplejo) y no el estado entusiasta que se esperaba.
- La correlación inversa entre la variable Sorpresa y la variable DIAT indica, que ante una marca conocida el consumidor se siente tranquilo y confiado, pues asocia la misma con la idea de calidad. Por tanto, esta asociación provoca estados de ánimo positivos (placer) debido a la seguridad que aporta una marca conocida, pero no está provocando sorpresa.

\section{Conclusiones}

Todos los GIFs ofrecieron puntuaciones positivas en la Valencia y valores negativos en la variable Activación (Estudio 1), situándose en el cuarto cuadrante del modelo circumplejo. En este cuadrante el consumidor se halla en un estado de calma y relajación, ya que el neocortex disminuye su nivel de activación para ahorrar energía, al tiempo que el área cerebral correspondiente a las emociones cobra mayor importancia (Michael, Ramsoy, Stephens y Kotsi, 2019; Modica, et al., 2018).

Por otra parte, la visualización de los GIFs ofreció valores negativos para la variable Sorpresa, y el Estudio 2 mostró que los participantes asociaron de forma inconsciente marca conocida a calidad. Esto nos ha llevado a concluir que el

\section{Figura 4. Posicionamiento GIFs estudiados conforme Modelo Circumplejo. Russell (1980)}

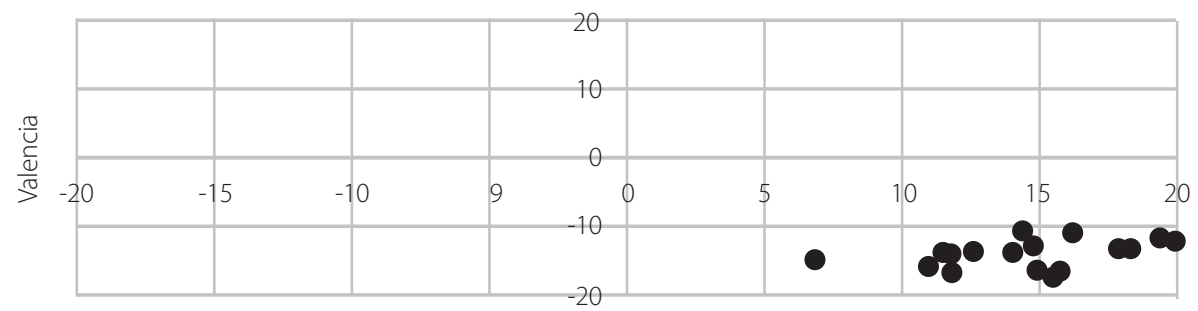


uso de GIFs en las estrategias de comunicación, al tratarse de una marca conocida por el usuario, no asegura situar al consumidor en un estado emocional entusiasta que le impulse hacia la compra. Aunque sí se esté generando un estado de placer y tranquilidad que se ha relacionado con la percepción implícita de calidad. Por tanto, los GIFs de marcas conocidas no están consiguiendo provocar sorpresa en los usuarios de las redes sociales, a pesar de considerar al GIF como un recurso innovador en sí mismo.

Se concluye que las marcas conocidas, para conseguir una respuesta emocional entusiasta, necesitan no solo usar recursos visuales innovadores, sino utilizarlos de forma sorprendente para situar a los usuarios en un estado emocional entusiasta (primer cuadrante del modelo Circumplejo de Russell, 1980). Este hecho se podría considerar como una paradoja de la fama.

Se ha mostrado que conseguir engagement de los usuarios no es suficiente para obtener éxito en las estrategias de comunicación digital de las marcas conocidas. Hay que dar un paso más e implicar activamente al consumidor sorprendiéndole con el diseño de los recursos utilizados. En el caso de los GIFs, el diseño de la interacción de la visualización (McKenna et al., 2017) podría ser una de las propuestas para conseguir GIFs que sorprendan al usuario.

\section{Limitaciones e implicaciones}

El método de investigación utilizado ha mostrado su utilidad para medir el engagement de los usuarios en la visualización de los GIFs; utilizando métricas como el signo y la intensidad de la emoción, y herramientas de neuromarketing.

Desde el principio se decidió limitar el estudio 1 a marcas consideradas como de gran valor (Interbrand,2017) para tener un objeto de estudio concreto que permitiera resultados significativos.
Sin embargo, el hecho de no haber podido comparar los efectos emocionales de GIFs de estas marcas tan populares con GIFs de marcas menos conocidas ha supuesto una limitación para las conclusiones del estudio. Por ello, consideramos que sería muy interesante poder realizar una nueva investigación con el mismo diseño metodológico, para observar el impacto emocional de GIFs de marcas menos populares.

Otra de las limitaciones del estudio ha sido el tener que grabar en un video los GIFs para poder realizar el diseño experimental. Esto ha supuesto una limitación a la interacción de los usuarios con los GIFs. A partir de la conclusión de que las marcas conocidas tienen que utilizar los recursos visuales digitales de forma sorprendente para activar a los consumidores, proponemos una investigación que compare los efectos emocionales generados por GIFs con diferentes diseños de interacción (Boy, Detienne, y Fekete, 2015). De llevarse a cabo esta investigación, se podría observar si alguno de los modelos de interacción provoca sorpresa; y cuál de estos diseños de la experiencia interactiva (MacKanna et al., 2017) conseguiría generar un mayor engagement, activando emociones de signo positivo mas intensas.

El estudio ha permitido mostrar que la utilización de formas nuevas de comunicación no siempre genera sorpresa en los usuarios. Es decir, las marcas renombradas no deben dar por sentado que, utilizando herramientas innovadoras en sus estrategias de comunicación, van a llamar la atención y motivar al usuario hacia la compra. Consideramos que esta implicación del estudio puede ser muy relevante para el diseño de GIFs. Por ello, nos proponemos realizar una nueva investigación que compare los efectos emocionales que ha provocado cada GIF, y determinar qué elementos comunes de diseño comparten los GIFs de mayor impacto emocional. 


\section{Bibliografía}

Aaker, David (1991). Managing brand equity. Capitalizing on the Value of a Brand Name. The Free Pres. New York.

Aaker, D. (1996). Measuring brand equity across products and markets. California management review, 38(3), 102-120 Adami, E. y Jewitt, C. (2016). Special issue: Social media and the visual. Visual Communication, 15(3), 263-270

Anderson, K. J. (1990). Arousal and the inverted-u hypothesis: A critique of Neiss's "Reconceptualizing arousal".

Atwal, G. y Williams, A. (2017). Luxury brand marketingthe experience is everything! In Advances in luxury brand management. Brand Management Vol. 16, 5/6, 338-346

Audrin, C., Brosch, T., Sander, D. y Chanal, J. (2018). More than meets the eye: the impact of materialism on information selection during luxury choices. Frontiers in behavioral neuroscience, 12,172

Bagozzi, R.; Gopinath, M. y Nyer, P. (1999). The role of emotions in marketing. Journal of the academy of marketing science, 27(2), 184-206.

Bellman, S., Wooley, B., y Varan, D. (2016). Program-ad matching and television ad effectiveness: A reinquiry using facial tracking software. Journal of Advertising, 45(1), 72-77.

Blanton, H., Jaccard, J., Gonzales, P. y Christie, Ch. (2006). Decoding the implicit association test: Implications for criterion prediction. Journal of Experimental Social Psychology, 42(2), 192-212.

Boy, J., Detienne, F. y Fekete, J.D. (2015). Storytelling in information visualizations: Does it engage users to explore data? In Proceedings of the 33rd Annual ACM Conference on Human Factors in Computing Systems. pp. 1449-1458.

Brakus, J., Schmitt, B., y Zarantonello, L. (2009). Brand experience: what is it? How is it measured? Does it affect loyalty? Journal of Marketing, 73: 52-68.

Brodie, R. J., Hollebeek, L. D., Jurić, B., y Ilić, A. (2011). Customer engagement: Conceptual domain,

fundamental propositions, and implications for research. Journal of service research, 14(3),

252-271

Burnett, M. S. y Lunsford, D. A. (1994) Conceptualizing guilt in the consumer decision-making process.Journal of Consumer Marketing, 11(3), 33-43
Cacioppo, S., Bolmont, M. y Monteleone, G. (2018). Spatio-temporal dynamics of the mirror neuronsystem during social intentions. Social neuroscience, 13(6), 718-738.

Calder, B. J., Malthouse, E. C. y Maslowska, E. (2016) Brand marketing, big data and social innovation as future research directions for engagement. Journal of Marketing Management, 32(5-6), 579-585

Chang, C. T., y Chen, P. C. (2017). Cause-related marketing ads in the eye tracker: it depends on how you present, who sees the ad, and what you promote. International Journal of Advertising, 36(2), 336-355.

Chang, Y., Ko, Y. J. y Carlson, B. D. (2018). Implicit and explicit affective evaluations of athlete brands: The associative evaluation-emotional appraisal-intention model of athlete endorsements. Journal of Sport Management, 32(6), 497 510 .

Chang, B. W., y Ungar, D. (1993, December). Animation from cartoons to the user interface. In Proceedings of the 6th annual ACM symposium on User interface software and technology (pp. 45-55)

Cingel, D. P. y Krcmar, M. (2014). Understanding the experience of Imaginary Audience in a social media environment. Journal of Media Psychology.

Ding, C. y Tseng, T. (2015). On the relationships among brand experience, hedonic emotions, and brand equity, European Journal of Marketing, Vol. 49 No. 7/8, pp. 994 1015.

Duffett, R. (2015). Facebook advertising's influence on intention-to-purchase and purchase amongst Millennials. Internet Research, 25(4), 498-526

Enli, G. (2017). Twitter as arena for the authentic outsider Exploring the social media campaigns of Trump and Clinton in the 2016 US presidential election. European journal of communication, 32(1), 50-61.

Ekman, P. (1993). Facial expression and emotion. American psychologist, 48(4), 384-392

Ekman, P., Freisen, W. V., y Ancoli, S. (1980). Facial signs of emotional experience. Journal of Personality and Social Psychology, 39(6), 1125-1134

Estrella, A., y Segovia, C. (2016). Comunicación Integrada de Marketing. Madrid: ESIC.

Fernández-Abascal, E., García, B., Jiménez, M.P., Martín, M.D., y Domínguez, F. (2010). Psicología de la Emoción. Madrid: Centro de estudios Ramón Areces, S.A 
Galmés, M. y Victoria, J (2012). La organización de eventos en el contexto de las Comunicaciones Integradas de Marketing (IMC): el valor de la experiencia. Pensar la Publicidad, 2012, vol. $6, n^{\circ} 1,15-34$.

Galmés, M. (2015) Comunicación y marketing experiencial: aproximación al estado de la cuestión. Opción,31(1),974-999. From https://www.redalyc.org/pdf/310/31043005054.pdf González-Sánchez, J.L., y Gil-Iranzo, R. M. (2013). Factores hedónicos y multiculturales que mejoran las experiencias de usuario en el diseño de productos. El profesional de la información, 22(1).

Gibson, B. (2008). Can evaluative conditioning change attitudes toward mature brands? new evidence from the implicit association test. Journal of Consumer Research, 35(1), 178-188. Godey, B., Manthiou, A., Pederzoli, D., Rokka, J., Aiello, G., Donvito, R., y Singh, R. (2016). Social mediamarketing efforts of luxury brands: Influence on brand equity and consumer behavior. Journal of business research, 69(12), 5833-5841.

Goyal, G. y Singh, J. (2018, April). Minimum Annotation identification of facial affects for Video Advertisement. In 2018 International Conference on Intelligent Circuits and Systems (ICICS) (pp. 300-305). IEEE.

Greenwald, A. y Banaji, M. (1995). Implicit social cognition: attitudes, self-esteem, and stereotypes. Psychological review, 102(1), 4-27

Greenwald, A. G., Nosek, B. A., y Banaji, M. R. (2003). Understanding and using the implicit associationtest: I. An improved scoring algorithm. Journal of personality and social psychology, 85(2), 197.

Guerreiro, J., Rita, P. y Trigueiros, D. (2015). Attention, emotions and cause-related marketing effectiveness. European Journal of Marketing, 49(11-12), 1728-1750.

Gürsimsek, Ö. (2016). Animated GIFs as vernacular graphic design: producing Tumblr blogs. Visual Communication, 15(3), 329-349.

Havlena, W. y Holbrook, M. B. (1986). The varieties of consumption experience: comparing two typologies of emotion in consumer behavior. Journal of consumer research, 13(3), 394-404.

He, S., Zheng, X., Zeng, D., Luo, C. y Zhang, Z. (2016). Exploring entrainment patterns of human emotion in social media. Plos one, 11(3).
Hirschman, E. y Holbrook, M. B. (1982). Hedonic consumption: emerging concepts, methods and propositions. Journal of marketing, 46(3), 92-101.

Hollebeek, L (2011) Exploring customer brand engagement: definition and themes, Journal of Strategic Marketing, 19:7, 555-573.

Holmes, Emily, M., Andrew, M., Bundy y Dalgleish, T. (2008). The causal effect of mental imagery on emotion assessed using picture-word cues. Emotion, 8(3), 395.

Interactive Advertising Bureau y Elogia (2018): Estudio anual de redes sociales 2018. IAB Spain. Versión reducida. Disponible en https://iabspain.es/estudio/estudio-anual-de-redes-sociales-2018/

Interbrand (2017): Interbrand releases 2017 Best Global Brands report. September 24, 2017 Disponible en https:// www.interbrand.com/newsroom/bgb-report-2017/

Izard, C. E. (1991). The Psychology of Emotions. Nueva York: Plenum Press.

Jaeger, S. R., Spinelli, S., Ares, G. y Monteleone, E. (2018). Linking product-elicited emotional associations and sensory perceptions through a circumplex model based on valence and arousal: Five consumer studies. Food research international, 109, 626-640.

Jou, B., Bhattacharya, S. y Chang, S. (2014, November). Predicting viewer perceived emotions in animated GIFs. In Proceedings of the 22nd ACM international conference on Multimedia. pp. 213-216.

Kafetsios, K., Chatzakou, D., Tsigilis, N. y Vakali, A. (2017). Experience of emotion in face to face and computer-mediated social interactions: An event sampling study. Computers in Human Behavior, 76, 287-293.

Kahneman, D. y Tversky, A. (1979). Prospect theory: An analysis of decision under risk. En Handbook of the fundamentals of financial decision making: Part I. 2013. p. 99-127.

Kantar Worldpanel (2018). Brand Footprint. A Global ranking of the most chosen consumer brands (6). Disponible en www.kantarworldpanel.com/brandfootprint

Klaus, P. y Maklan, S. (2007). The role of brands in a service-dominated world. Journal of Brand Management, 15(2), 115-122.

Lang, P. J. (1995). The Emotion Probe: Studies of Motivation and Attention. American Psychologist. 50, 372-385. 
Lavidge, R. y Steiner, G. (1961). A model for predictive measurements of advertising effectiveness. Journal of marketing, 25(6), 59-62

Likowski, K. U., Mühlberger, A., Gerdes, A., Wieser, M. J., Pauli, P. y Weyers, P. (2012). Facial mimicry and the mirror neuron system: simultaneous acquisition of facial electromyography and functional magnetic resonance imaging. Frontiers in human neuroscience, 6, 214-

Maison, D., Greenwald, A. G. y Bruin, R. H. (2004). Predictive validity of the implicit association test in studies of brands, consumer attitudes, and behavior. Journal of Consumer Psychology, 14(4), 405-415

McDuff, D., El Kaliouby, R. y Picard, R. W. (2012). Crowdsourcing facial responses to online videos. IEEE Transactions on Affective Computing, 3(4), 456-468.

McKenna, S.; Henry Riche, N.; Lee, B.; Boy, J.; Meyer, M. (2017). "Visual narrative flow: Exploring factors shaping data visualization story reading experiences". Eurographics conference on visualization (EuroVis), v. 36, n. 3, pp. 377 387.

Michael, I., Ramsoy, T., Stephens, M., y Kotsi, F. (2019). A study of unconscious emotional and cognitive responses to tourism images using a neuroscience method. Journal of Islamic Marketing, 10(2), 543-564.

Miltner, K. M. y Highfield, T. (2017). Never gonna GIF you up: Analyzing the cultural significance of the animated GIF. Social Media y Society, 3(3).

Modica, E., Cartocci, G., Rossi, D., Martínez, A. C., Cherubino, P., Maglione, A. G., . . Babiloni, F. (2018). Neurophysiological responses to different product experiences. Computational Intelligence and Neuroscience, Volume 2018, Article ID 9616301, 10 pages.

Monge-Benito, S. y Fernández-Guerra, V. (2011). Neuromarketing: Tecnologías, Mercado y Retos. Pensar la publicidad, 5(2), 19-25.

Mundel, J., Huddleston, P., Behe, B., Sage, L. y Latona, C. (2018). An eye tracking study of minimally branded products: Hedonism and branding as predictors of purchase intentions. Journal of Product and Brand Management, 27(2), 146-157.

Novak, T., Hoffman, D. y Yung, Y. (2000). Measuring the Customer Experience in Online Environments: A Structural Modeling Approach. Marketing Science, 19(1), 22-42.
Öhman, A. (1987). Psychophysiology of emotion: An evolutionary cognitive perspective. En T.K. Ackles, J.R. Jennings y M.G.H. Coles (Eds.), Advances in Psychophysiology, volumen 2, 79-127.

Ophuis, P. y Van Trijp, H. (1995). Perceived quality: A market driven and consumer oriented approach. Food quality and Preference, 6(3), 177-183.

Pawle, J. y Cooper, P. (2006). Measuring emotion-Lovemarks, the future beyond brands. Journal of advertising research, 46(1), 38-48.

Plutchik, R. E. y Conte, H. R. (1997). Circumplex models of personality and emotions. American Psychological Association.

Powers, T., Advincula, D., Austin, M. S., Graiko, S., y Snyder, J. (2012). Digital and social media in the purchase decision process: A special report from the Advertising Research Foundation. Journal of advertising research, 52(4), 479-489.

Prescott, J. (2017). Some considerations in the measurement of emotions in sensory and consumer research. Food Quality and Preference, 62, 360-368.

Reimann, M., Castano, R. Zaichkowsky, J. y Bechara, A (2012). How we relate to brands: Psychological and neurophysiological insights into consumer-brand relationships. Journal of Consumer Psychology, 22(1), 128-142.

Russell, J. A. (1980). A circumplex model of affect. Journal of Personality and Social Psychology, 39(6), 1161-1178.

Ryan, R. M. y Deci, E. L. (2001). On happiness and human potentials: A review of research on hedonic and eudaimonic well-being. Annual review of psychology, 52(1), 141-166.

Schmitt, B. (1999). Experiential marketing. Journal of marketing management, 15(1-3), 53-67.

Schmitt, B., Brakus, J. y Zarantonello, L. (2015). From experiential psychology to consumer experience. Journal of Consumer Psychology, 25(1), 166-171.

Scolari, C. (2012). Comunicación Digital. Recuerdos de futuro. El Profesional de la Información. 2012; 21 (4): 337-340 Sharma, N. (2018). Decoding the effects of a product's cast shadow in brand advertising. Journal of Product and Brand Management, 27(2), 103-114.

Skandalis, A., Byrom, J. y Banister, E. (2019). Experiential marketing and the changing nature of extraordinary experiences in post-postmodern consumer culture. Journal of Business Research, 97, 43-50. 
Smith, A. K. y Bolton, R. N. (2002). The effect of customers' emotional responses to service failures on their recovery effort evaluations and satisfaction judgments. Journal of the academy of marketing science, 30(1), 5-23.

Tapia Frade, A., Martín Guerra, E. y Puente, J. E. (2016). Neurociencia y publicidad. Atención, emoción y su relación con los premios obtenidos en el Festival Internacional de Publicidad de Cannes. Anàlisi: quaderns de comunicació i cultura, 0075-95.

Tianliang, L., Junwei, W., Xiubin, D., Feng L., Quanzeng, Y. y Jiebo L. (2019). Sentiment Recognition for Short Annotated GIFs Using Visual-Textual Fusion. IEEE Transactions on Multimedia, vol. 22, no. 4, pp. 1098-1110, April 2020. 10.1109/TMM.2019.2936805.

Thurlow, C., Aiello, G. y Portmann, L. (2019). Visualizing teens and technology: A social semiotic analysis of stock photography and news media imagery. New Media y Society. 2020, Vol. 22(3) $528-549$.

Tolins, J. y Samermit, P. (2016). GIFs as embodied enactments in text-mediated conversation. Research on Language and Social Interaction, 49(2), 75-91.

Vecchiato, G., Fallani, F. D. V., Astolfi, L., Toppi, J., Cincotti, F., Mattia, D., ... y Babiloni, F. (2010). The issue of multiple univariate comparisons in the context of neuroelectric brain mapping: an application in a neuromarketing experiment. Journal of neuroscience methods, 191(2), 283-289.

Vila, J., Ramírez, I., Sánchez, M. y Fernández-Santaella, M.

C. (2001). El Sistema Internacional de Imágenes Afectivas
(IAPS): Adaptación española. Revista de psicología general y aplicada, 08-21.

Vriens, M., Chen, S. y Schomaker, J. (2019). The evaluation of a brand association density metric. Journal of Product and Brand Management, 28(1), 104-116.

Walla, P., Koller, M., Brenner, G. y Bosshard, S. (2017). Evaluative conditioning of established brands: Implicit measures reveal other effects than explicit measures. Journal of Neuroscience Psychology and Economics, 10(1), 24-41.

Weibel, D., di Francesco, R., Kopf, R., Fahrni, S., Brunner, A., Kronenberg, P., . . . y Wissmath, B. (2019). TV vs. YouTube: TV advertisements capture more visual attention, create more positive emotions and have a stronger impact on implicit long-term memory. Frontiers in Psychology, 10, 626.

Yoo, B. y Donthu, N. (2001). Developing and validating a multidimensional consumer-based brand equity scale. Journal of business research, 52(1), 1-14.

Yuang, Y. H. E. y Wu, C. K. (2008). Relationships among experiential marketing, experiential value, and customer satisfaction. Journal of Hospitality y Tourism Research, 32(3), 387-410.

Zeithaml, V. (1988). Consumer perceptions of price, quality, and value: a means-end model and synthesis of evidence. Journal of marketing, 52(3), 2-22.

Zhang, J. y Mao, E. (2016). From Online Motivations to Ad Clicks and to Behavioral Intentions: An Empirical Study of Consumer Response to Social Media Advertising. Phsychology y Marketing, 33(3), 155- 164. 
